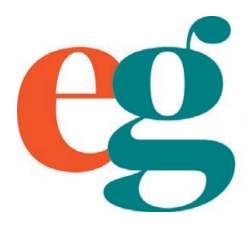

\title{
Masculinidades, desigualdad social y embarazo en varones adolescentes mexicanos
}

\section{Masculinities, Social Inequality, and Pregnancy in Mexican Adolescent Males}

\section{Olga Lorena Rojas}

El Colegio de México, Ciudad de México, México. email: olrojas@colmex.mx

(1) https://orcid.org/0000-0002-5374-5378

Recibido: 20 de mayo

2021

Aceptado: 12 de agosto de 2021

Publicado: 30 de agosto de 2021

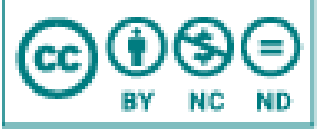

Esta obra está protegida bajo una Licencia Creative Commons Atribución-NoComercial-

SinDerivadas 4.0 Internacional (CC BY-NC-ND 4.0]

\section{Resumen}

En este artículo ponemos en cuestionamiento las afirmaciones que señalan al embarazo durante la adolescencia como precursor de la pobreza, del abandono escolar y de la incorporación laboral temprana entre la población adolescente involucrada. Para ello, desde una perspectiva que toma en cuenta el género, la desigualdad social y el curso de vida, revisamos los resultados de la investigación existente sobre el tema en México y analizamos el particular caso masculino. Argumentamos que los embarazos en la etapa adolescente son el resultado de una serie de desventajas sociales que los jóvenes varones acumulan en su precoz tránsito a la vida adulta.

Palabras clave: género; desigualdad social; embarazo adolescente; jóvenes varones, México.

CÓMO CITAR: Rojas, Olga Lorena. (2021). Masculinidades, desigualdad social y embarazo en varones adolescentes mexicanos. Revista Interdisciplinaria de Estudios de Género de El Colegio de México, 7, e817. doi: http://dx.doi.org/10.24201/reg.v7i1.817

http://estudiosdegenero.colmex.mx 


\begin{abstract}
In this essay, we question the statements that point to pregnancy during adolescence as a precursor of poverty, school dropout and early entry into the workforce among the adolescent population involved. By considering gender, social inequality and life course, we review the results of existing research on the subject in Mexico and analyze the male case in particular. We argue that teenage pregnancies arise from a set of social disadvantages accumulated by young men in their early transition to adult life.
\end{abstract}

Key words: gender, social inequality, teenage pregnancy, young men, Mexico.

\title{
Introducción
}

El embarazo y la fecundidad durante la adolescencia son fenómenos persistentes en México, puesto que sus altas tasas se han mantenido relativamente estables a través del tiempo y las generaciones (Giorguli y Angoa, 2013; Sánchez y Pérez, 2016). Sin embargo, llama la atención que recientemente se esté observando un incremento, puesto que, si en 2009 se registraron 69 nacimientos por cada mil mujeres de 15 a 19 años, para 2014 este indicador alcanzó los 77.4 nacimientos (Conapo, 2016). Este comportamiento contrasta al compararlo con la constante disminución observada en las tasas de fecundidad de los demás grupos de edad (Welti, 2005). Estos datos generaron preocupación en algunas instancias del gobierno mexicano, de tal suerte que en 2015 se puso en marcha la Estrategia Nacional para la Prevención del Embarazo en Adolescentes (ENAPEA).

En este marco, es importante señalar la existencia de significativas diferencias en las tasas de fecundidad adolescente al considerar la persistente desigualdad social en el país. Diversos estudios han señalado que el embarazo y los nacimientos entre la población adolescente ocurren con mayor frecuencia en estratos sociales empobrecidos, que tienen escasos niveles de escolaridad y muy precarias oportunidades de empleo (Menkes y Suárez, 2003; Solís, Gayet y Juárez, 2008; Stern y Menkes, 2008; Welti, 2005). 
En esos grupos sociales prevalecen todavía valoraciones muy tradicionales sobre la sexualidad, la vida en pareja y la reproducción. Es frecuente que las y los jóvenes procreen a sus primeros hijos de manera muy próxima a su iniciación sexual, durante la adolescencia, sin haber utilizado algún método anticonceptivo (Amuchástegui, 2001; Menkes y Suárez, 2003; Szasz, 2008).

Por ello, se suele atribuir al embarazo adolescente la reproducción de la pobreza, porque se considera que limita las posibilidades de que las mujeres obtengan una mayor escolarización, lo que a su vez impide que puedan obtener un empleo calificado y reduce su acceso a recursos que permitan un desarrollo adecuado para sus hijos. Se señala entonces que este tipo de embarazos podría perpetuar la pobreza de estas jóvenes y sus familias como un círculo vicioso (Welti, 2000).

Un informe reciente del Fondo de Población de las Naciones Unidas (UNFPA), titulado "Consecuencias socioeconómicas del embarazo en la adolescencia en seis países de América Latina y el Caribe", en el que se incluye el caso mexicano, señala que el embarazo en la adolescencia y la maternidad temprana impactan de manera determinante en la trayectoria de vida de miles de mujeres en América Latina, puesto que lastra su vida y reproduce la pobreza. De acuerdo con este estudio, las niñas y las adolescentes que son madres suelen abandonar la escuela para criar a sus hijos, lo que se traduce en una mayor dificultad para continuar sus estudios y encontrar un empleo bien remunerado. Por ello, estas jóvenes mujeres terminan dedicándose exclusivamente a las tareas domésticas y tienen menos oportunidades de desarrollarse. Se concluye entonces que el embarazo adolescente es una "fábrica de pobres" (La Jornada, 2020).

Sin embargo, los hallazgos de diversas investigaciones realizadas en el país ponen en cuestionamiento este tipo de argumentos, puesto que en los grupos sociales de escasos recursos económicos la mayoría de las y los adolescentes que habían tenido un hijo abandonaron la escuela un año antes de convertirse en madres y padres (Giorguli y Angoa, 2013; Sánchez y Pérez, 2016). 
Además, se ha constatado que, en estos mismos estratos sociales pobres, el primer empleo de los varones casi siempre antecede al evento reproductivo y a la vida conyugal, mientras que para las mujeres el nacimiento del primer hijo y la unión conyugal preceden al primer empleo (Pérez, 2012; Sánchez y Pérez, 2016). En contraste, el embarazo durante la adolescencia en estratos sociales medios y altos es mucho menos frecuente y, en caso de ocurrir, se registra en edades más tardías y cuando las adolescentes todavía se encuentran estudiando. Además, una importante proporción de estas jóvenes se embaraza antes de unirse (Stern y Menkes, 2008).

En este contexto, es importante mencionar que, asociadas al fenómeno del embarazo durante la adolescencia, las uniones tempranas también se han mantenido relativamente constantes a través del tiempo en el país (Giorguli y Angoa, 2013).

En efecto, no todos los adolescentes involucrados en procesos de embarazo se encuentran en soltería, puesto que se ha observado que más de la mitad de los padres y madres adolescentes se encontraban unidos, consensualmente o en matrimonio, un año antes de tener a su primer hijo. De hecho, la entrada en unión constituye un factor de riesgo muy importante que propicia y precipita los embarazos, así como la entrada a la maternidad y la paternidad durante la adolescencia (Giorguli y Angoa, 2013; Sánchez y Pérez, 2016).

Por ello, no resulta extraño saber que es bastante común que las mujeres adolescentes de los estratos sociales más desfavorecidos no consideren que sus embarazos constituyan un problema, en particular si se encuentran unidas o casadas. En cambio, entre la población adolescente con mejores condiciones socioeconómicas, se ha observado que es más probable que manifiesten que los embarazos no sean deseados e incluso que se interrumpan (Menkes y Suárez, 2003, 2013).

Puede decirse entonces, de acuerdo con los resultados de diversos estudios realizados en el país, que la gran mayoría de los embarazos durante el periodo de la adolescencia ocurre en grupos sociales empobrecidos, en el marco de una unión conyugal (generalmente de tipo consensual), de la actividad laboral de los varones involucrados, quienes, al igual que sus compañeras, han 
abandonado el sistema escolar (Menkes y Suárez, 2003; Sánchez y Pérez, 2016; Solís, Gayet y Juárez, 2008; Stern y Menkes, 2008).

En este artículo deseamos reflexionar y aportar evidencias y argumentos para afirmar que en los grupos sociales precarios y empobrecidos, el abandono del sistema escolar, la temprana incorporación masculina al mercado laboral y la unión temprana de las parejas son condiciones que facilitan la ocurrencia de los embarazos durante la adolescencia y no al contrario. Es decir, el embarazo adolescente no es una "fábrica de pobres", como comúnmente se señala. Sostenemos que el embarazo en la adolescencia no es la causa, sino el resultado de la acumulación de diversas desventajas sociales en la trayectoria de vida de los adolescentes en su precoz tránsito a la vida adulta.

En este trabajo nos proponemos analizar, desde una perspectiva de género, que contempla la desigualdad social y el curso de vida, la presencia de los varones en los procesos de embarazo durante la adolescencia. Para ello, en la primera parte del trabajo aportamos elementos relativos al proceso de conformación de la identidad de género masculina, enfocándonos en particular en dos eventos cruciales: la iniciación sexual y la incorporación al mundo laboral como mandatos de género que contribuyen a la construcción de la masculinidad. En la segunda parte, damos cuenta desde la perspectiva de curso de vida — en particular, desde el análisis de trayectorias y transiciones de vida - del proceso de acumulación de desventajas sociales que experimentan los varones adolescentes de los grupos más desfavorecidos del país en su pasaje a la adultez y que propician los embarazos durante su adolescencia. Utilizar esta perspectiva en particular nos permite observar con mayor claridad la secuencia de las transiciones de vida de los adolescentes y poner en evidencia la falsa causalidad atribuida a los embarazos en la adolescencia respecto a la reproducción de la pobreza, al abandono escolar y la temprana incorporación laboral de los adolescentes. Concluimos esta reflexión con algunas consideraciones finales en las que revisamos el papel que desempeñan los contextos familiares en los procesos de embarazo durante la adolescencia. 


\section{El proceso de conformación de la identidad de género masculina}

Desde la perspectiva de género, la conformación de la identidad masculina está relacionada con un conjunto de disposiciones que configuran la manera de pensar, las percepciones e interpretaciones acerca de la vida individual y social, además de los marcos de socialización, los sentimientos y las prácticas de los hombres. La interiorización de la manera en que se es hombre depende de la cultura y de la sociedad de que se trate. Los varones aprenden a ser masculinos a partir de la existencia de una cultura de género (Bourdieu, 1991, 2000).

La conformación de la identidad de género masculina es un fenómeno plural, puesto que el modelo hegemónico de masculinidad no siempre puede ser seguido por todos los hombres. Existen variaciones en función del grupo social y cultural, la etnia, la edad y la generación a las que pertenezcan los varones (Amuchástegui, 2001; Olavarría, 2000; Salguero, 2007; Szasz, 1998a, 2008).

Las identidades masculinas son recreadas en la actuación cotidiana de los varones, no están fijas ni acabadas, se pueden modificar en la práctica y en el curso de la historia particular de cada individuo. Se van construyendo y reajustando a través de las diferentes etapas de la vida y en los distintos contextos sociales y culturales en donde se sitúen los hombres (Salguero, 2007).

A pesar de la existencia de una diversidad de identidades masculinas, existen al mismo tiempo semejanzas notables entre los hombres que comparten ciertas experiencias socioculturales e históricas. Gran parte de la reproducción del sistema de género tiene lugar a través de las actividades rutinarias y las interacciones de la vida diaria en los espacios familiar, escolar, laboral y público. Estos ámbitos de socialización, desde la infancia hasta la vida adulta, son los lugares desde donde se interiorizan los significados, los valores y los marcos de interpretación y de relacionamiento social (Gutmann, 2000).

Cuando se habla de la conformación de la identidad masculina, o de la masculinidad, se está haciendo referencia a un proceso que tiene lugar en la vida de los hombres y que está centrado 
en la búsqueda permanente de prestigio social y de reafirmación constante de los atributos masculinos (Szasz, 2008). Estos atributos están relacionados fundamentalmente con el ejercicio de la sexualidad, el trabajo remunerado, la proveeduría y la reproducción. En este proceso, los hombres buscan cumplir con determinadas pruebas establecidas socialmente y observadas por su grupo de pares. Por medio de la realización de estas prácticas y experiencias —que en muchas ocasiones se tornan en ritos de pasaje - los hombres van conformando su propia imagen como individuos masculinos adultos que paulatinamente se separan e independizan de la protección de sus progenitores (Amuchástegui, 2001; Núñez, 2013; Olavarría, 2002; Rojas, 2019; Szasz, 2008).

Algunos discursos sobre los estereotipos masculinos están relacionados con el honor, la reputación, la fortaleza, la virilidad, la valentía, el riesgo y el ocultamiento de las emociones y los sentimientos, desde los cuales se elaboran significados y representaciones acerca de ser hombre. Esta imagen brinda respeto a su portador y también proporciona seguridad a su familia (Salguero, 2007, 2008).

Desde esta perspectiva, el paso a la vida adulta desde la experiencia masculina está relacionado con ciertos eventos como el comienzo de la actividad sexual, la salida del sistema escolar, el ingreso al mundo laboral, el inicio de una unión conyugal y la procreación de los hijos. En términos sociales, los varones que logran cumplir y superar este conjunto de transiciones consiguen reconocimiento y prestigio como hombres que han completado su tránsito a la adultez (Olavarría, 2000, 2002).

En efecto, haberse iniciado sexualmente, realizar un trabajo remunerado y procrear un hijo, constituyen pruebas irrefutables del tránsito masculino a la vida adulta que han de superarse para poner fin a la etapa de la juventud. Con ellas se asumen nuevas responsabilidades relacionadas con la posibilidad de conformar una unión conyugal y de procrear una descendencia, pero, sobre todo, con la capacidad de mantener a una familia que depende de la proveeduría masculina (Amuchástegui, 2001; Módena y Mendoza, 2001; Núñez, 2013; Olavarría, 2000). 
Sin embargo, la temporalidad de entrada a la adultez y la forma de acceder a ella guardan mucha relación con los recursos materiales y culturales de la familia de origen, además de las oportunidades que brindan los contextos económico, social y comunitario. Estas condiciones, fuertemente marcadas por la desigualdad social, regulan de manera diferencial para cada estrato o grupo social la experiencia de esta transición vital (Oliveira y Mora, 2008a, 2008b; Rojas, 2019; Solís, 2016).

Por ello, en una sociedad tan desigual en términos sociales y económicos como la mexicana, si se quieren comprender las diferentes configuraciones que asume el pasaje a la vida adulta entre los varones — que necesitan terminar de conformar su identidad genérica masculina- y observar las particularidades que la estratificación social imprime a esta importante transición en la vida de los hombres, es imprescindible tomar en cuenta la intersección existente entre el género y la desigualdad social. Consideramos que es precisamente en este contexto que los procesos de embarazo durante la adolescencia adquieren un papel significativo, puesto que con frecuencia forman parte de este tránsito vital e identitario de los varones de estratos sociales precarios, como lo veremos más adelante (Stern, 2007; Stern y Menkes, 2008).

\section{La importancia de iniciarse sexualmente}

En la medida en que la pertenencia al mundo masculino requiere ser reafirmada y demostrada constantemente, una de las formas para lograrlo es el desempeño sexual. La actividad sexual de los hombres es una de las principales formas de representación y reafirmación de su identidad de género, puesto que por medio de ella se expresa y se mide el poder masculino y se marcan sus límites. El control de la sexualidad masculina todavía se rige por valores culturales, normas de género, discursos sobre la masculinidad, controles comunitarios y familiares, e incluso por las presiones de los grupos de pares (Amuchástegui, 2001; Hernández-Rosete, 2006; Szasz, 1998a).

El inicio de la vida sexual en diversas culturas implica un suceso de gran importancia en el paso de la adolescencia a la adultez porque señala el momento en que los adolescentes y jóvenes alcanzan las condiciones que les hacen capaces de incorporarse a la vida adulta. La manera en 
que los hombres dan sentido a esta experiencia tiene lugar en un contexto cultural que brinda ciertos marcos de interpretación (Amuchástegui, 2001; Dixon-Mueller, 1996).

Las percepciones y las prácticas sexuales están profundamente moldeadas por el ámbito social y cultural en el cual las vidas de los jóvenes se desenvuelven. Por ello, para entender las implicaciones de esta importante transición es vital considerar las características del contexto en el que ocurre (Binstok y Gogna, 2015; Menkes, De Jesús y Sosa, 2019; Stern, 2007; Zhu y Bosma, 2019).

En el particular espacio simbólico de la sexualidad, los varones encuentran la posibilidad de reafirmar su masculinidad en su tránsito a la adultez. Por ello, en este pasaje al mundo adulto, adquieren mucha importancia tanto la iniciación de su vida sexual, como la constante demostración de su actividad heterosexual ante los demás, sobre todo ante el grupo de pares (Amuchástegui, 2001; Kaufman, 1997; Olavarría, 2004).

Diversas investigaciones sobre los significados de la sexualidad entre la población mexicana reportan que existe un doble patrón moral marcado por la diferencia de género que define las características de las experiencias sexuales de hombres y mujeres, particularmente en lo que se refiere a la iniciación sexual. En el caso masculino la iniciación sexual adquiere un carácter ritual, socialmente organizado por hombres de mayor experiencia, quienes proveen la oportunidad y las condiciones propicias para que el joven se inicie sexualmente y pueda ser aceptado como miembro del grupo de hombres (Amuchástegui, 2001; De Jesús, 2011; Szasz, 1998a).

La virginidad de los varones después de cierta edad despierta sospechas pues constituye un signo de una masculinidad dudosa ${ }^{1}$. Por eso en la iniciación sexual masculina, la presión del

\footnotetext{
${ }^{1}$ En contraste, la vigilancia sobre la sexualidad femenina difiere respecto al caso masculino puesto que para ellas se afirma el alto valor que tiene la virginidad y la importancia de preservarla hasta la unión matrimonial (Amuchástegui, 2001; Módena y Mendoza, 2001). El inicio de la sexualidad femenina se encuentra estrechamente vinculado a un compromiso afectivo, al inicio de una vida en pareja y de la procreación del primer hijo. Las relaciones sexuales prematrimoniales entre las mujeres adquieren legitimidad solamente en el contexto de una relación amorosa y de un proyecto de pareja. Este conjunto de valoraciones y normatividades sociales dificultan el uso de anticoncepción cuando las mujeres adolescentes y jóvenes inician su vida sexual (Menkes y Suárez, 2004; Módena y Mendoza, 2001; Szasz, 1997, 2001).
} 
grupo social juega un papel de considerable importancia pues está orientada a la confirmación de la identidad masculina y a la regulación de las prácticas sexuales del joven (Amuchástegui, 2001). Este vínculo entre desempeño sexual e identidad de género influye en la permisividad social hacia diversas prácticas sexuales masculinas que incluyen el abuso sexual y la imposición de relaciones sexuales (Szasz, 2001).

Los resultados de algunos estudios realizados en el país confirman que los comportamientos sexuales declarados por los varones son claramente diferentes de los reportados por las mujeres. Los hombres mexicanos inician su actividad coital heterosexual a una edad más temprana que las mujeres, en promedio entre los 15 y los 17 años. Ellos señalan haber tenido diversas experiencias sexuales previas a la unión conyugal y mayoritariamente con parejas con las que no establecieron una relación afectiva (con una amiga o una desconocida). En su experiencia la primera relación sexual coital y la unión conyugal ocurren en momentos diferentes, separados por varios años (Menkes, De Jesús y Sosa, 2019; Szasz, 1998b).

Al respecto, conviene señalar que en nuestro país se han observado modelos sociales y culturales de comportamiento sexual y reproductivo diferenciados, no solamente entre hombres y mujeres, sino también entre distintos estratos sociales. Estos modelos se caracterizan no solamente por claras diferencias temporales en el inicio de la actividad sexual, el primer embarazo, la primera unión y el nacimiento del primer hijo, sino también por la elección de la persona con la que se comienza la vida sexual. En los estratos medios y altos los varones jóvenes se inician sexualmente con amigas o con sus novias en mayor proporción que aquellos de estratos sociales bajos (Oliveira y Mora, 2008a).

En particular, cuando se ha investigado sobre el comienzo de la vida sexual de hombres adolescentes y jóvenes de estratos sociales populares urbanos y de ámbitos rurales, se ha encontrado que estos varones se construyen socialmente como sujetos sexuales a partir del modelo predominante de masculinidad, en el cual se requiere demostrar que se es sexualmente activo y, además, que se es heterosexual para ser considerado un hombre de verdad. El espacio de la sexualidad constituye una de las dimensiones más importantes a partir de la cual los hombres reafirman su identidad de género ante sí mismos y frente a su grupo social. Por ello, 
es común que el inicio sexual masculino se encuentre marcado por una fuerte presión social ejercida por el grupo de pares e incluso por los familiares (Amuchástegui, 2001; De Jesús, 2011; Núñez, 2007; Stern, 2012).

Entre los varones de estos grupos sociales el comienzo de la vida sexual es una experiencia necesaria para perder la ingenuidad infantil y adquirir saberes necesarios para hacerse hombres. El inicio precoz de la actividad sexual y que ésta sea frecuente confirma su adscripción al mundo adulto masculino. Por ello, esta importante transición se experimenta como un ritual de pasaje que, en algunos casos, se lleva a cabo con mujeres consideradas por los jóvenes varones como "expertas" o incluso con prostitutas. En otros casos, el noviazgo constituye una oportunidad para iniciarse sexualmente y para entrenarse en esta materia, por ello, algunos varones ejercen presión sobre sus parejas para tener relaciones sexuales. En cualquier caso, el debut sexual cobra relevancia porque a partir de esta experiencia, los jóvenes son aceptados socialmente por su grupo de amistades (Amuchástegui, 2001; De Jesús, 2011; Núñez, 2007; Stern, 2007).

Debido a que el primer encuentro sexual se experimenta bajo condiciones de mucha presión social y espontaneidad, los jóvenes de estos sectores sociales son impulsivos y toman riesgos, por ello, no utilizan métodos anticonceptivos, ya sea para prevenir un embarazo o la transmisión de alguna infección sexual (De Keijzer, 1998; De Jesús, 2011).

Una vez que se han iniciado sexualmente, es común que en sus subsecuentes intercambios sexuales los jóvenes varones de estos grupos sociales mantengan la diferenciación entre las mujeres con quienes se relacionan. Por lo general, clasifican a las mujeres en dos grupos, por un lado, se encuentran las mujeres que consideran inocentes, decentes y valiosas para ser sus novias o para conformar con ellas una unión, porque tienen un comportamiento serio y no manifiestan malicia ni tener conocimientos o deseos sexuales. Son consideradas respetables y confiables, es decir, "mujeres de su casa" que no amenazan la honorabilidad de su hombría porque son vírgenes cuando se inician con ellos sexualmente (Núñez, 2007). Con ellas no usan ningún tipo de protección o método anticonceptivo porque las consideran puras. Lo anterior pone de manifiesto una masculinidad que se construye mediante la apropiación de la sexualidad 
femenina, puesto que los varones tienen la garantía de que ellos son los únicos depositarios de los deseos sexuales femeninos ${ }^{2}$ (Amuchástegui, 2001; Arias y Rodríguez, 1998; De Jesús, 2011; Núñez, 2007; Rojas, 2020; Stern, 2007).

Y, por otro lado, se encuentran las mujeres erotizadas y que manifiestan activamente sus deseos sexuales, consideradas promiscuas y poco confiables con las que no buscarán tener un noviazgo o formalizar una unión. Los jóvenes consideran que de este tipo de mujeres deben cuidarse ante la posibilidad de contraer una infección de transmisión sexual. Por esta razón, con estas parejas sexuales tienden a usar el condón masculino como protección para ellos (Amuchástegui, 2001; Arias y Rodríguez, 1998; De Jesús, 2011; Núñez, 2007; Rojas, 2020; Stern, 2007).

En cualquiera de los dos casos se constatan los prejuicios de estos jóvenes varones en materia sexual respecto a las mujeres, puesto que, en su opinión, son ellas quienes pueden transmitir las infecciones sexuales y son también quienes se embarazan. Además, se aprecia que entre los varones existe una fuerte disociación entre el ejercicio de su sexualidad y su capacidad reproductora, que se traduce en la negación de su responsabilidad fecunda durante las relaciones sexuales. Este conjunto de valoraciones masculinas respecto a la sexualidad y la reproducción constituyen fuertes obstáculos para el uso del condón masculino como método anticonceptivo para prevenir los embarazos durante la adolescencia y la juventud temprana de los varones (Amuchástegui, 2001; Arias y Rodríguez, 1998; De Jesús, 2011; Rojas, 2020; Stern, 2007).

\footnotetext{
${ }^{2}$ Entre las mujeres de estos grupos sociales desfavorecidos todavía permanece una fuerte normativa de género que establece una necesaria cercanía entre la iniciación sexual y la vida en pareja, puesto que la sexualidad femenina solo es válida en el contexto de la unión conyugal y de la maternidad, elementos que continúan definiendo la vida y la valoración social de estas mujeres. Por ello, la práctica anticonceptiva femenina en estos grupos sociales es fuertemente rechazada (Amuchástegui, 2001; Bellato, 2001, 2006; Módena y Mendoza, 2001; Szasz, 2001, 2008). La permanencia de esta norma social fomenta la persistencia del embarazo adolescente como parte de la trayectoria de vida de estas mujeres, marcada por una unión muy temprana y seguida de —o precedida por - un embarazo (Menkes y Suárez, 2003, 2004; Stern, 1997, 2007; Stern y Menkes, 2008).
} 


\section{La importancia de incorporarse al mundo laboral para dejar la etapa juvenil y demostrar que se es capaz de proveer}

Entre los diversos atributos que distinguen a los hombres como tales, el cumplimiento del papel como proveedores tiene un carácter estructurador, puesto que permite distanciarse de la etapa juvenil y de la protección paterna, al tiempo que se adquiere la aptitud para conformar y mantener una familia. Asumir la responsabilidad de la proveeduría es particularmente importante entre aquellos varones que se han unido y que son padres, es decir, que han formado una familia que depende de ellos (Fuller, 1997; Olavarría, 2000; Núñez, 2013).

Por ello, el trabajo extradoméstico remunerado es un componente sustantivo de la identidad de género masculina puesto que otorga sentido y continuidad al proyecto de vida, al tiempo que permite conseguir reconocimiento social, familiar e individual. La ocupación, es decir, el trabajo por el que se obtiene un ingreso es uno de los factores determinantes del prestigio y del lugar que ocupan los hombres en la sociedad (Olavarría, 2000, 2002; Rosas 2007; Salguero, 2008).

La identidad masculina se acredita de manera importante a partir de la autosuficiencia económica y del estatus social que se obtiene del empleo remunerado. Además, el cumplimiento del papel de proveedor se encuentra asociado al ejercicio de poder y autoridad en el hogar, puesto que quien provee puede controlar los ingresos y decidir su manejo (Olavarría, 2001).

Las representaciones sociales respecto a la importancia del mundo del trabajo se van incorporando durante el proceso de socialización familiar y escolar de los varones, formando parte de la subjetividad e identidad masculinas. La aspiración que los hombres tienen de incorporarse a un trabajo remunerado se encuentra vinculada a la obtención de un lugar en el mundo público, a partir del cual serán reconocidos socialmente. No cumplir con esta meta o prueba significa no estar a la altura de las expectativas sociales (Pérez, 2012; Salguero, 2007).

En el caso mexicano, diversas investigaciones, desde aproximaciones de corte cualitativo y cuantitativo, han dado cuenta de que el inicio de la actividad laboral es una de las transiciones 
más importantes en la vida de los hombres, porque con ello adquieren la capacidad de cumplir con una de las expectativas sociales y culturales de género fundamentales, que es el cumplimiento de su papel como proveedores de sus hogares (Núñez, 2007; Pérez, 2012).

Se trata de un cambio fundamental sin el cual no pueden ser considerados hombres adultos. El ingreso al mercado laboral (formal o informal) es parte indisoluble de la identidad de género masculina que asegura el pasaje a la adultez, por ello, esta imprescindible transición no puede dejar de realizarse ni ser pospuesta en la trayectoria de vida de los varones. Entre los jóvenes mexicanos se trata de una transición temprana, tanto en su calendario como en su intensidad, puesto que casi todos los varones terminan por incorporarse relativamente pronto al mercado de trabajo. Prácticamente todos los hombres en su juventud buscan insertarse en alguna actividad económica, aunque se trate de empleos precarios y de muy baja remuneración (Módena y Mendoza, 2001; Núñez, 2007, Pérez, 2012).

La importancia que adquiere para los hombres mexicanos el cumplimiento del rol de proveedor de sus hogares se manifiesta en el hecho de que el $82 \%$ del total de los varones urbanos se convierte en el principal sostén económico de sus familias antes de cumplir los 31 años (Martínez y Ferraris, 2016).

El trabajo remunerado ${ }^{3}$ es un factor central en las vidas de los hombres - de cualquier estrato o grupo social - porque les permite adquirir el sentimiento de hombría, con el cual ya pueden aspirar a ser esposos y padres, puesto que ya son capaces de mantener un hogar. Con el empleo y los ingresos que de él se obtienen, los hombres adquieren importancia y valor social (Bellato, 2001; Módena y Mendoza, 2001; Núñez, 2007; Rojas, 2008).

En este sentido, resulta importante destacar que diversas investigaciones de corte cualitativo llevadas a cabo en el país reportan que, de acuerdo con el modelo de masculinidad dominante, los hombres que se consideran adultos se caracterizan porque trabajan de manera remunerada,

\footnotetext{
${ }^{3}$ En contraste, para las mujeres mexicanas el trabajo remunerado no se ha generalizado como una parte importante de su desarrollo y proyecto de vida, puesto que los marcadores sociales y de género establecen para ellas el matrimonio y la maternidad como transiciones preferentes para ingresar a la vida adulta y obtener reconocimiento social (Módena y Mendoza, 2001; Pérez, 2012).
} 
constituyen una familia, tienen hijos, son la autoridad y los proveedores del sustento en sus hogares. Por ello, para los varones la paternidad es un evento de gran trascendencia puesto que significa dos cosas fundamentales: conformar un hogar que depende de ellos y asumir, mediante el trabajo remunerado, la responsabilidad de asegurar el bienestar material familiar (Bellato, 2001; Módena y Mendoza, 2001; Rojas, 2008).

Los hijos son una marca de distinción que separa y jerarquiza a los varones, puesto que un hombre debe ser capaz de responsabilizarse de ellos. La paternidad es un mecanismo que comprueba física y moralmente los atributos masculinos ante los demás, al constituirse en una vía para hacerse personas responsables. El nacimiento de los hijos otorga sentido a la vida personal, conyugal y laboral de los varones. La conformación de una familia constituye un deber ser en el cual ubican trascendencia, obligación, responsabilidad, racionalidad, adultez y su propia realización (Bellato, 2001; Módena y Mendoza, 2001; Rojas, 2008).

Es importante señalar que la investigación relativa al proceso en el que los hombres mexicanos se convierten en proveedores ha reportado diferencias sociales y económicas, puesto que en los estratos sociales más precarios se transita más temprano a la vida laboral con un escaso capital humano porque, generalmente, han abandonado los estudios de manera precoz. En contraste, en los estratos sociales acomodados, los hombres cuentan con condiciones favorables para llevar a cabo de manera más pausada su incorporación al mercado de trabajo con la finalidad de prolongar su permanencia en el sistema escolar (Pérez, 2012; Solís, 2016).

Estas diferencias por estrato social se observan claramente en el hecho de que los varones pertenecientes a estratos sociales bajos se convierten en proveedores aproximadamente a los 22 años, en tanto que aquellos de estratos sociales medios y altos asumen esta responsabilidad dos años más tarde en promedio. Estos últimos tardan más en experimentar esta transición porque tienen un calendario más tardío para la entrada en unión y la formación de sus familias (Martínez y Ferraris, 2016). 


\section{Acumulación de desventajas sociales en el tránsito a la vida adulta y los embarazos durante la adolescencia de los varones de estratos sociales empobrecidos}

De acuerdo con la perspectiva de curso de vida, las trayectorias de las personas forman parte de, y están moldeadas por, su situación social y el tiempo histórico en el que se desenvuelven sus vidas y que ofrecen diversas posibilidades a partir de las cuales los individuos van construyendo su existencia y toman determinadas decisiones (Elder, 1985; Tuirán, 1999).

Las experiencias tempranas y el significado que se les otorga generan nuevas situaciones y oportunidades. El impacto de un evento o de una transición está definido por el contexto de la biografía de las personas (que es la suma de eventos vividos), los ámbitos en los que se experimentaron, significados atribuidos y la forma como serán asimilados en la trayectoria del curso de vida. Las trayectorias de vida son experimentadas de manera interdependiente puesto que las influencias sociales e históricas se expresan a través de las redes de relaciones de las personas. A su vez, las personas no viven sus existencias aisladamente, ya que la familia, la comunidad, los amigos, los compañeros de la escuela, entre otros, pueden influir en sus acciones y decisiones (Elder, 1985).

Además, la posición de clase brinda recursos sociales, económicos y oportunidades, así como esquemas culturales y mentales que permiten tener una visión particular del mundo y de los comportamientos deseables, creando un conjunto de aspiraciones de referencia. Por ello, las biografías de los individuos se encuentran definidas por una concatenación particular de condiciones materiales de existencia, una configuración de transiciones en el curso de vida y determinados esquemas mentales que les otorgan sentido (Tuirán, 1999).

En particular, el pasaje a la vida adulta es un proceso que abarca un periodo del curso de vida de las personas y está moldeado por diversas instituciones como la escuela, la familia, el mercado laboral, la religión y el Estado. En general, se considera que el tránsito a la vida adulta se encuentra marcado por una trayectoria que implica un cambio de roles de la juventud hacia los que se consideran propios de la adultez (Elder, 1985; Tuirán, 1999). 
En este pasaje al mundo adulto la desigualdad social moldea diferentes trayectorias porque se traduce en diferencias, tanto en las oportunidades que tienen los jóvenes para este tránsito, como en la forma y el momento en que experimentan los diversos eventos que marcan su entrada a la adultez (Oliveira y Mora, 2008a).

Sin embargo, este pasaje al mundo adulto está marcado no solamente por la desigualdad socioeconómica, sino también por las normativas y las valoraciones de género que regulan las acciones y las maneras de pensar de las personas a partir de la interacción en diversos espacios e instituciones sociales, tales como la familia, la escuela, la comunidad, el mercado de trabajo. El conjunto de prescripciones culturales de género marca las pautas sobre los comportamientos masculinos y femeninos esperados y deseables (Szasz, 2008). El tránsito a la adultez no escapa al control de estas estructuras culturales que evalúan las acciones y los logros de las personas de acuerdo con su género, al tiempo que confieren prestigio y estima social o, por el contrario, estigmas sociales (Amuchástegui, 2001).

En la investigación relativa al curso de vida de adolescentes y jóvenes pertenecientes a estratos sociales empobrecidos se observa sistemáticamente la existencia de diversas desventajas sociales que afectan sus procesos de transición a la vida adulta y, por consiguiente, sus trayectorias de vida. En el ámbito del hogar una desventaja importante se relaciona con condiciones precarias de sobrevivencia y privaciones, una mayor proporción de personas dependientes, relaciones autoritarias y el frecuente uso de la violencia como mecanismo para resolver conflictos en la familia. En conjunto estas situaciones pueden configurar importantes desventajas sociales que afectan las trayectorias de vida juveniles (Alvarado, 2014; Azaola, 2004; Mora y Oliveira, 2014; Nava, 2014).

Por ello, se señala que, en el proceso de transición a la vida adulta, la desigualdad social, la pobreza y la precariedad resultan de fundamental trascendencia en la definición de las trayectorias futuras de los jóvenes, puesto que constituye un periodo en el que se cristalizan las desigualdades de oportunidades que dejan huella profunda - muchas veces definitoria - en las decisiones y los acontecimientos futuros de sus biografías (Mora y Oliveira, 2009, 2014; Thomson, Bell, Holland, Herderson, McGellis y Sharpe, 2002). 
La violencia cotidiana como forma de resolución de conflictos y la falta de convivencia de los hijos con sus padres (generalmente ausentes) configuran contextos familiares hostiles para el desarrollo de los jóvenes, muy comunes en los sectores sociales económicamente precarios. Este tipo de ambientes familiares puede contribuir a definir las trayectorias de vida de los jóvenes en las que acumulan varias desventajas que propician frecuentemente transiciones precoces a la vida adulta (Mora y Oliveira, 2014; Nurius, Prince y Rocha, 2015; Schafer, Ferraro y Mustillo, 2011; Schoon y Melis, 2019; Vandecasteele, 2010).

En estos contextos sociales precarios y marginados es común que los adolescentes y jóvenes deban asumir roles adultos de manera anticipada durante su adolescencia, abandonando la escuela e incorporándose a empleos manuales no calificados. La ocurrencia de estas transiciones a edades tempranas contribuye a reproducir las desigualdades sociales y la precariedad a lo largo de sus trayectorias vitales. En particular, su ingreso precoz al mercado de trabajo - en empleos manuales no calificados - define de manera decisiva sus futuras decisiones y trayectorias de vida. Cuando estos adolescentes y jóvenes asumen responsabilidades familiares de manera temprana su tránsito a la adultez se anticipa y acelera. Se convierten en adultos en plena etapa adolescente o juvenil (Alcázar, Balarín, Glave y Rodríguez, 2020; Mora y Oliveira, 2014; Rodríguez, 2001; Wagmiller y Adelman, 2009).

En el caso mexicano la investigación social de tipo cualitativo, que se ha enfocado en el tránsito a la vida adulta de los hombres de estratos sociales empobrecidos, ha dado cuenta de que ante la imposibilidad de obtener prestigio o reconocimiento social por medio de un buen empleo o una elevada escolaridad, estos varones intentan dar prueba de su identidad de género de manera precoz, si se les compara con sus pares de estratos acomodados, quienes tienen las condiciones propicias para llevar a cabo de manera más pausada y reflexiva su tránsito a la adultez. Para los hombres de estratos bajos se hace necesario probar tempranamente que son capaces de tener actividad sexual, de reproducirse mediante la procreación de un hijo, de obtener un empleo, de conformar un vínculo conyugal y, sobre todo, de conformar y mantener a una familia (Amuchástegui, 2001; Módena y Mendoza, 2001; Rojas, 2008; Szasz, 2001). 
En este contexto, es necesario destacar que, a pesar de los avances conseguidos en el país respecto a la expansión educativa y al incremento en el nivel promedio de instrucción, se observa que el calendario de la salida de la escuela es notoriamente diferente entre los jóvenes mexicanos según el estrato socioeconómico al que pertenezcan. Para los jóvenes de estratos sociales bajos, dejar la escuela es una transición claramente más temprana si se les compara con aquellos de estratos medios y altos (Solís, 2016; Solís, Gayet y Juárez, 2008). La prematura salida del sistema escolar de los jóvenes varones de sectores sociales empobrecidos — por lo general entre los 13 y 14 años en promedio- se relaciona frecuentemente con una incorporación temprana a empleos de baja calificación y en condiciones muy precarias (Giorguli, 2005; Giorguli y Angoa, 2013; Solís, 2016).

Cuando se estudian las trayectorias de los jóvenes varones que han tenido un hijo durante la adolescencia, se ha observado que la mayoría de ellos ya había dejado la escuela un año antes de convertirse en padres (Giorguli y Angoa, 2013; Sánchez y Pérez, 2016).

El abandono del sistema escolar entre estos jóvenes de estratos sociales más pobres está muy relacionado con un precoz inicio de la actividad laboral, en tanto constituye una de las transiciones más importantes en la vida masculina, puesto que con ello se está en capacidad de cumplir con las expectativas sociales y culturales de género. Estos muchachos transitan más temprano a la vida laboral respecto a sus pares de otros estratos sociales y lo hacen con un escaso capital humano, incorporándose por lo general a empleos de baja remuneración (Mora y Oliveira, 2014; Oliveira y Mora, 2008b; Pérez, 2012).

Son la pobreza y la escasez de recursos que sufren sus familias de origen las que llevan a estos jóvenes a abandonar la escuela precozmente y a ingresar de manera temprana al mercado laboral (casi siempre informal) para colaborar en la manutención de sus hogares. Muchos de estos jóvenes han experimentado el abandono del padre y difícilmente logran concluir la educación secundaria. Al experimentar estas transiciones de manera tan prematura, estos jóvenes acumulan claras desventajas sociales que marcarán el rumbo de sus futuras trayectorias vitales (Mora y Oliveira, 2014; Rojas, 2019, 2020). 
Sin embargo, pese a que sus empleos son precarios y sus ingresos escasos, el trabajo remunerado detona en ellos un cambio fundamental en sus vidas, puesto que les confiere la percepción de que ya son hombres adultos que pueden ser considerados capaces de responder, de mantener y, por lo tanto, de formar una familia que puede depender de ellos. Para ellos, tener un empleo remunerado constituye una importante prueba de hombría que les permitirá recibir aprobación y reconocimiento social (Rojas, 2019, 2020).

No es extraño entonces que diversas investigaciones hayan dado cuenta de que casi todos los hombres adolescentes de estos estratos sociales, involucrados en procesos de embarazo durante su adolescencia, ya contaban con una actividad remunerada al menos un año antes de tener a su primer hijo, en promedio entre los 14 y los 16 años (Giorguli y Angoa, 2013; Pérez, 2012; Sánchez y Pérez, 2016). Esta temprana transición laboral guarda estrecha relación con su precoz salida de la escuela y no parece modificarse a lo largo del tiempo, puesto que se mantiene así a través de las generaciones (Martínez y Ferraris, 2016; Pérez, 2012).

Entre los muchachos de estos estratos sociales es común que una vez que se han incorporado al mercado de trabajo muestren mayor propensión a dejar la casa familiar (Oliveira y Mora, 2008a). Sin embargo, en algunos casos estos jóvenes continúan viviendo con sus padres porque, en tanto proveedores, contribuyen de manera importante a la manutención de su familia. Además, también es muy común que en estos estratos sociales empobrecidos la unión y la formación temprana de la familia no siempre lleve a la conformación de hogares independientes. La escasez de recursos económicos de estas nuevas y jóvenes parejas les obliga a compartir la vivienda con la familia de alguno de los miembros de la pareja, sobre todo durante los primeros años de unión (Giorguli y Angoa, 2013; Oliveira y Mora, 2008a; Sánchez y Pérez, 2016).

En efecto, entre estos varones de estratos sociales bajos se observa un repunte de las trayectorias que involucran el tránsito a la vida en pareja y el mantenimiento de la corresidencia con los padres o suegros por un tiempo. Estos jóvenes ya han abandonado la escuela y se han incorporado al empleo remunerado, sin embargo, frente a las dificultades económicas para tener independencia residencial, inician la vida en pareja y la conformación de su descendencia en la 
vivienda familiar. En estos ámbitos sociales es notoria la centralidad y la solidaridad de la familia de origen en el pasaje a la vida adulta de estos varones (Solís, 2016).

En este contexto, cuando se analizan las transiciones relacionadas con la entrada en unión, el primer embarazo y el nacimiento del primer hijo entre los varones jóvenes de estos estratos sociales más desaventajados, se observa que estos eventos se encuentran fuertemente vinculados al comienzo de su actividad sexual. Estos hallazgos guardan relación con aquellos que confirman que los jóvenes de estos grupos sociales tienden a unirse, embarazar a su pareja y tener hijos a edades más tempranas que los jóvenes de estratos sociales medios y altos (Oliveira y Mora, 2008a).

Es relevante señalar que, en el estudio particular de la ocurrencia de los embarazos durante la adolescencia, se ha observado sistemáticamente que la mayoría de los padres adolescentes vivía en pareja (a los 17 años en promedio) un año antes de tener a su primer hijo. Además, este comportamiento se ha mantenido en el tiempo (Giorguli y Angoa, 2013; Sánchez y Pérez, 2016).

En este sentido, los hallazgos de algunos estudios cualitativos, centrados en los relatos de varones adolescentes de estratos sociales desfavorecidos, han reportado que los entrevistados señalaron, de manera generalizada, que los embarazos de sus novias o compañeras fueron muy esperados y deseados. De acuerdo con sus testimonios, el embarazo durante la adolescencia no se percibe ni se experimenta como un trastorno que haya impedido su desarrollo vital ${ }^{4}$. Por el contrario, para ellos este suceso contribuyó a reafirmar su imagen como hombres cabales y responsables. Además, el embarazo de sus parejas y el eventual nacimiento de sus hijos, contribuyeron a fortalecer la unión con su pareja y a conformar una familia que depende de ellos económicamente. De hecho, los adolescentes mencionaron el deseo explícito de la paternidad como principal medio para transitar a la adultez y consolidar un proyecto de vida (De Jesús, 2011; Rojas, 2019, 2020).

\footnotetext{
${ }^{4}$ Noción que se corresponde con los hallazgos que reportan que es común entre las mujeres adolescentes de los estratos sociales más desfavorecidos — muchas de las cuales se encuentran unidas - considerar que sus embarazos no son un problema, puesto que son deseados y esperados (Menkes y Suárez, 2003, 2013).
} 
Para estos varones, la paternidad es una experiencia fundamental puesto que se transforma en un motor que otorga sentido y dirección a sus vidas. En los contextos precarios en los que transcurre su vida, el nacimiento de sus hijos se convierte en una fuerte motivación para desempeñarse laboralmente, así como para superarse social y económicamente. Con este encadenamiento de eventos, valoraciones y motivaciones, que culmina con el nacimiento del primer hijo, estos jóvenes varones refuerzan de manera importante la percepción de que han adquirido una masculinidad completa (Rojas, 2019, 2020).

\section{Consideraciones finales}

En este trabajo de reflexión hemos podido constatar que los embarazos en la etapa adolescente en su gran mayoría son producto de la pobreza y la escasez de recursos en las que se desarrollan las vidas de los jóvenes involucrados y no al contrario, como comúnmente se piensa. De hecho, constituyen el resultado de la acumulación de una serie de desventajas sociales en el tránsito hacia la etapa adulta de estos hombres.

La permanencia de los embarazos en la adolescencia a través del tiempo y de las generaciones en los estratos sociales más desfavorecidos en el país, permite afirmar que se trata de un fenómeno que tiene una profunda raíz en la desigualdad social, que configura formas de ser hombre claramente diferenciadas. Por ello, es necesario llamar la atención sobre la importancia que adquiere, para su análisis y comprensión, considerar la intersección entre el género y la desigualdad social (Rojas, 2019, 2020).

Los embarazos durante la adolescencia forman parte de las trayectorias de vida de los jóvenes varones de estratos sociales de escasos recursos, que están marcadas por transiciones a la adultez a edades más tempranas que sus pares de estratos sociales mejor posicionados. Estos jóvenes anticipan y aceleran su tránsito a la etapa adulta llevados por la precariedad familiar, el menor acceso a oportunidades escolares $\mathrm{y}$, muy frecuentemente, porque han tenido que asumir responsabilidades familiares de manera precoz. En este contexto, los eventos que definen y confirman su paso a la adultez $-\mathrm{y}$ que se transforman en desventajas sociales que van 
acumulando - adquieren para ellos significados diferentes y particulares en función de su posición social (Mora y Oliveira, 2014; Oliveira y Mora, 2008a, 2008b).

Estos jóvenes buscan probar su masculinidad de manera temprana mediante la actividad sexual que, eventualmente, contribuirá a dar prueba de su virilidad con la fecundación (Szasz, 1998a). En estas experiencias sexuales iniciales prevalece entre ellos la negación de su potencial fecundo, que los lleva a evitar el uso de algún método anticonceptivo, incluido el condón. De acuerdo con sus concepciones, dado que los embarazos ocurren en el cuerpo de las mujeres, corresponde a ellas la responsabilidad de evitarlos. Por ello, el embarazo precoz, durante su adolescencia, es un evento que materializa una determinada forma de pensar y de actuar sobre la sexualidad (Rojas, 2019, 2020).

En estos contextos sociales empobrecidos — en los que los logros escolares y laborales son escasos-, los jóvenes varones enfrentan fuertes dificultades para encontrar un lugar y una posición que les permitan obtener reconocimiento social, por ello, los procesos de embarazo y, sobre todo, la paternidad durante la adolescencia, se experimentan como una forma de trascendencia social que, a la vez, acredita la entrada a la adultez. Esto explica que lejos de ser considerados como un problema, los embarazos en esta etapa constituyen un elemento que estructura y consolida una particular forma de completar la identidad de género masculina en estos estratos sociales. En esta temprana experiencia vital, las redes familiares constituyen un factor que contribuye a reproducir estas concepciones y prácticas (De Jesús, 2011; Rojas, 2019, 2020).

Se trata de un particular patrón de tránsito a la vida adulta que se ha mantenido en el país a lo largo del tiempo, puesto que no muestra cambios a través de las generaciones y que ocurre en un periodo relativamente corto en la vida de los jóvenes varones de estos grupos sociales, por lo general, de los 11 a los 18 años. En este breve lapso experimentan precipitadamente todas las diferentes transiciones del pasaje a la adultez, siendo adolescentes todavía, en secuencias que comienzan con una iniciación sexual precoz y la prematura salida de la escuela, continúan con el ingreso temprano al trabajo (frecuentemente en el sector informal), seguido de la unión en pareja (generalmente de tipo consensual) y el nacimiento del primer hijo. Se ha observado 
de manera sistemática que entre estos varones el abandono del sistema escolar y el primer empleo anteceden al nacimiento del primer hijo y al comienzo de la vida en unión. Lo anterior confirma que la alta valoración que tiene para ellos el cumplimiento de la proveeduría para conseguir reconocimiento social como hombres responsables (De Jesús, 2011; Sánchez y Pérez, 2016; Rojas, 2020).

Es importante destacar que, aunque las vidas de estos jóvenes parecen estar marcadas por la precariedad y la adversidad, los contextos familiares a los que pertenecen conforman importantes redes de solidaridad. Estas redes constituyen un importante apoyo económico y emocional durante los procesos de embarazo y los nacimientos de los hijos de estos jóvenes varones (Rojas, 2019, 2020).

La pertenencia a este tipo de ambientes familiares favorece el compromiso, la cooperación, el apoyo mutuo y la primacía de los intereses del grupo antes que los individuales, todos ellos valores colectivistas que caracterizan al familismo mexicano y que aún persiste en amplios sectores sociales, sobre todo entre aquellos de contextos precarios. En estos grupos sociales el tejido comunitario y familiar se consolida y fortalece mediante la conformación de obligaciones y deudas mutuas a lo largo del ciclo de vida familiar (Robichaux, 2006).

La pobreza que caracteriza la vida cotidiana de las familias de escasos recursos hace necesario el establecimiento de vínculos solidarios y de reciprocidad entre las generaciones. Las relaciones de intercambio de bienes y de trabajo entre sus integrantes, constituyen importantes principios culturales y estrategias de sobrevivencia en estos grupos solidarios familiares (Robichaux, 2006).

Estas configuraciones familiares particulares son las que acompañan y construyen alrededor de los embarazos de sus miembros adolescentes una red de sustento y contención emocional importante. En estos ámbitos familiares los jóvenes consiguen protección material, un importante reconocimiento y la confirmación social como hombres adultos y responsables. Todos estos elementos son fundamentales para entender la permanencia de los embarazos 
durante la adolescencia a través de las generaciones en estos contextos familiares precarios (Rojas, 2019, 2020).

Por ello, las directrices de las políticas públicas interesadas en la atención de este fenómeno deberían tomar en cuenta la persistente desigualdad social que caracteriza a nuestra sociedad y reconocer la existencia de una diversidad de significados de género atribuidos a los comportamientos sexuales y reproductivos de la población adolescente (Rojas, 2019, 2020).

Además de ello, es necesario llamar la atención sobre la escasez de información respecto a la población masculina involucrada en los embarazos y la fecundidad durante la adolescencia. Consideramos que cualquier estrategia gubernamental encaminada a prevenir estos fenómenos tendría que considerar la necesidad de generar información cuantitativa (encuestas sociodemográficas) y cualitativa sobre la participación de los varones en el embarazo adolescente, puesto que actualmente la información disponible se refiere de manera preponderante a la población femenina.

\section{Referencias bibliográficas}

Alcázar, Lorena; Balarín, María; Glave, Cristina y Rodríguez, Ma. Fernanda. (2020). Fractured lives: understanding urban youth vulnerability in Perú. Journal of Youth Studies, 23(2),140-159. https://doi.org/10.1080/13676261.2019.1587154

Alvarado, Arturo. (Ed.) (2014). Violencia juvenil y acceso a la justicia en América Latina, Vol. II. México: El Colegio de México.

Amuchástegui, Ana. (2001). Virginidad e iniciación sexual en México. Experiencias y significados. México: The Population Council, EDAMEX.

Arias, Rosario y Rodríguez, Marisela. (1998). A puro valor mexicano. Connotaciones del uso del condón en hombres de la clase media de la ciudad de México. En Susana Lerner (Ed.), 
Varones, sexualidad y reproducción (pp. 319-339). México: El Colegio de México, SOMEDE.

Azaola, Elena. (2004). Juventud: exclusión y violencia. Desacatos, (14), 7-11.

Bellato, Liliana. (2001). Representaciones sociales y prácticas de hombres y mujeres mazahuas sobre la sexualidad y la reproducción (Tesis de Maestría en Antropología). México: Centro de Investigaciones y Estudios Superiores en Antropología Social, CIESAS, México.

Bellato, Liliana. (2006). Representaciones de hombres mazahuas sobre su sexualidad. "Si tuviera relaciones diario, bigotes por todos lados”, En Juan Guillermo Figueroa, Lucero Jiménez y Olivia Tena (Eds.), Ser padres, esposos e hijos: prácticas y valoraciones de varones mexicanos (pp. 159-194). México: El Colegio de México.

Binstock, Georgina y Gogna, Mónica. (2015). La iniciación sexual entre mujeres de sectores vulnerables en cuatro provincias argentinas. Sexualidad, Salud y Sociedad. Revista Latinoamericana, (20),113-140.

Bourdieu, Pierre. (1991). El sentido práctico. Madrid: Taurus.

Bourdieu, Pierre. (2000). La dominación masculina. Barcelona: Anagrama.

Consejo Nacional de Población, CONAPO. (2016). Situación de la salud sexual y reproductiva. República Mexicana. México: CONAPO.

De Jesús, David. (2011). Adolescencias escindidas: sexualidad y reproducción adolescente en contextos urbano-marginales de Nuevo León. México: Universidad Autónoma de Nuevo León. 
De Keijzer, Benno. (1998). La masculinidad como factor de riesgo. En Esperanza Tuñón (Coord.), Género y salud en el sureste de México (pp. 197-219). Villahermosa: El Colegio de la Frontera Sur, ECOSUR, Universidad Autónoma de Tabasco.

Dixon-Mueller, Ruth. (1996). The Sexuality Connection in Reproductive Health. En Sondra Zeidenstein y Kirsten-Moore (Eds.), Learning about Sexuality: A Practical Beginning (pp. 137-157). Nueva York: The Population Council, International Women's Health Coalition.

Elder, Glenn. (1985). Life course dynamics: trajectories and transitions, 1968-1980. Ithaca: Cornell University Press.

Fuller, Norma. (1997). Identidades masculinas. Lima: Pontificia Universidad Católica del Perú.

Giorguli, Silvia. (2005). Deserción escolar, trabajo adolescente y trabajo materno en México. En Marta Mier y Terán y Cecilia Rabell (Coords), Jóvenes y niños. Un enfoque sociodemográfico (pp. 167-202). México: IIS-UNAM, FLACSO-México, Miguel Ángel Porrúa.

Giorguli, Silvia y Angoa, Adela. (2013). El tránsito a la adultez en tiempos de incertidumbre. Coyuntura Demográfica, 4, 39-44.

Gutmann, Matthew. (2000). Ser hombre de verdad en la ciudad de México. Ni macho ni mandilón. México: El Colegio de México.

Hernández-Rosete, Daniel. (2006). La vida extramarital masculina en tiempos de VIH/Sida. Usos y prácticas entre algunos varones con profesiones ligadas a las ciencias sociales. En Juan Guillermo Figueroa, Lucero Jiménez y Olivia Tena (Eds.), Ser padres, esposos e hijos: prácticas y valoraciones de varones mexicanos (pp. 195-217). México: El Colegio de México. 
Kaufman, Michael. (1997). Las experiencias contradictorias del poder entre los hombres. En Teresa Valdés y José Olavarría (Coords.), Masculinidad: Poder y crisis (pp. 63-81). Santiago: FLACSO.

La Jornada. (11 noviembre de 2020). Embarazo adolescente, "fábrica de pobres en AL": ONU. Recuperado de https://www.jornada.com.mx/ultimas/sociedad/2020/11/11/embarazoadolescente-201cfabrica-de-pobres-en-al201d-onu-4125.htm1

Martínez, Mario y Ferraris, Sabrina. (2016). Trabajo y masculinidad: el rol de proveedor en el México metropolitano. En Marie-Laure Coubès, Patricio Solís y María Eugenia Zavala (Coords.), Generaciones, cursos de vida y desigualdad en México (pp. 403-427). México: El Colegio de México, El Colegio de la Frontera Norte.

Menkes, Catherine, De Jesús, David y Sosa, Itzel. (2019). Jóvenes en México: ¿existen diferencias entre hombres y mujeres en su inicio sexual y uso de condón? Papeles de Población, 25(100), 183-210.

Menkes, Catherine y Suárez, Leticia. (2003). Sexualidad y embarazo adolescente en México. Papeles de Población, 9(35), 233-262.

Menkes, Catherine y Suárez, Leticia. (2004). Prácticas sexuales y reproductivas de las jóvenes mexicanas. En Emma Liliana Navarrete (Coord.), Los jóvenes ante el siglo XXI (pp. 19-43). México: El Colegio Mexiquense.

Menkes, Catherine y Suárez, Leticia. (2013). El embarazo de los adolescentes en México: ¿es deseado? Coyuntura Demográfica, 4, 21-28.

Módena, María Eugenia y Mendoza, Zuanilda. (2001). Géneros y generaciones. Etnografía de las relaciones entre hombres y mujeres de la ciudad de México. México: The Population Council, EDAMEX. 
Mora, Minor y de Oliveira, Orlandina. (2009). Los jóvenes en el inicio de la vida adulta: Trayectorias, transiciones y subjetividades. Estudios Sociológicos, XXVII(79), 267-290.

Mora, Minor y de Oliveira, Orlandina. (2014). Los caminos de la vida: acumulación, reproducción o superación de las desventajas sociales en México. Revista Mexicana de Ciencias Políticas y Sociales, LIX(20), 81-116.

Nava, Araceli. (2014). Los jóvenes, la violencia y la policía: el caso de Cancún. En Arturo Alvarado (Ed.), Violencia Juvenil y Acceso a la Justicia en América Latina (pp. 21-62). México: El Colegio de México.

Núñez, Guillermo. (2007). Vínculo de pareja y hombría: “Atender y mantener” en adultos mayores del Río Sonora, México. En Ana Amuchástegui e Ivonne Szasz (Coords.), Sucede que me canso de ser hombre...Relatos y reflexiones sobre hombres y masculinidades en México (pp. 141-184). México: El Colegio de México.

Núñez, Guillermo. (2013). Hombres sonorenses. Un estudio de género de tres generaciones. México: Pearson.

Nurius, Paula; Prince, Dana y Rocha, Anita. (2015). Cumulative Disadvantage and Youth WellBeing: A Multi-Domain Examination with Life Course Implications. Child Adolescent Social Work Journal, 32(6), 567-576. doi:10.1007/s10560-015-0396-2

Olavarría, José. (2000). Ser padre en Santiago de Chile. En Norma Fuller (Ed.), Paternidades en América Latina (pp. 129-174). Lima: Pontificia Universidad Católica del Perú.

Olavarría, José. (2001). Invisibilidad y poder. Varones de Santiago de Chile. En Mara Viveros, José Olavarría y Norma Fuller, Hombres e identidades de género. Investigaciones desde América Latina (pp. 153-264). Bogotá: Universidad Nacional de Colombia. 
Olavarría, José. (2002). Hombres: identidades, relaciones de género y conflictos entre trabajo y familia. En José Olavarría y Catalina Céspedes (Eds.), Trabajo y familia: ¿conciliación? Perspectivas de género (pp. 53-76). Santiago: Servicio Nacional de la mujer, SERNAM, FLACSO, Centro de Estudios de la Mujer, CEM.

Olavarría, José. (2004). La invisibilidad de los hombres en la sexualidad y la reproducción y sus consecuencias en la responsabilidad. Notas para el debate. En La fecundidad en América Latina: ¿Transición o revolución? (pp. 289-311). Santiago: CEPAL, CELADE.

Oliveira, Orlandina de y Mora, Minor. (2008a). Desigualdades sociales y transición a la adultez en el México contemporáneo. Papeles de Población, 14(57), 117-152.

Oliveira, Orlandina de y Mora, Minor. (2008b). Las diversas formas de hacerse adulto en México: Diferencias de clase y género a principios del Siglo XXI. En Ana María Tepichin (Coord.), Género en contextos de pobreza (pp. 35-55). México: El Colegio de México.

Pérez, Fabiola. (2012). Desigualdades de género en el inicio de la vida laboral estable. Papeles de Población, 18(72), 213-246.

Robichaux, David. (2006). Hogar, familia y grupos de acción: el sistema familiar mesoamericano ante los desafíos del siglo XXI. En Rosario Esteinou (Ed.), Fortalezas y desafíos de las familias en dos contextos: Estados Unidos de América y México (pp. 485517). México: Centro de Investigaciones y Estudios Superiores en Antropología Social, CIESAS.

Rodríguez, Jorge. (2001). Vulnerabilidad y grupos vulnerables: un marco de referencia conceptual mirando a los jóvenes. En Serie Población y Desarrollo, vol. 17, CELADE, CEPAL. 
Rosas, Carolina. (2007). Migrar para proveer. Cardaleños desde Veracruz a Chicago: Un estudio cualitativo con varones adultos. En Lucero Jiménez y Olivia Tena (Coords.), Reflexiones sobre masculinidad y empleo (pp. 473-506). México: CRIM, UNAM.

Rojas, Olga. (2008). Paternidad y vida familiar en la Ciudad de México. México: El Colegio de México.

Rojas, Olga. (2019). Masculinidad y desigualdad social: una dupla que puede explicar el embarazo adolescente en México. Otros Diálogos, (9), octubre-diciembre. Recuperado de https://otrosdialogos.colmex.mx/masculinidad-y-desigualdad-social-una-dupla-quepuede-explicar-el-embarazo-adolescente-en-mexico

Rojas, Olga. (2020). El tránsito a la vida adulta en la adversidad. En Fabiola Pérez y Ángeles Sánchez (Coords.), Los claroscuros del embarazo, la maternidad y la paternidad en la adolescencia. Un enfoque cualitativo (pp. 137-158). México: UNAM, Orfila.

Salguero, Alejandra. (2007). El significado del trabajo en las identidades masculinas. En Lucero Jiménez y Olivia Tena (Coords.), Reflexiones sobre masculinidad y empleo (pp. 429-448). México: CRIM, UNAM.

Salguero, Alejandra. (2008). Ni todo el poder ni todo el dominio: identidad en los varones, un proceso de negociación entre la vida laboral y familiar. En Juan Carlos Rodríguez y Griselda Uribe (Coords.), Masculinidades el juego de género de los hombres en el que participan las mujeres (pp. 247-268). México: Plaza y Valdés.

Sánchez, Ángeles y Pérez, Fabiola. (2016). De maternidades y paternidades en la adolescencia. En Marie-Laure Coubès, Patricio Solís y María Eugenia Zavala (Coords.), Generaciones, cursos de vida y desigualdad social en México (pp. 109-137). México: El Colegio de México, El Colegio de la Frontera Norte. 
Schafer, Markus; Ferraro, Keneth y Mustillo, Sarah. (2011). Children of Misfortune: Early Adversity and Cumulative Inequality in Perceived Life Trajectories. American Journal of Sociology, 116(4), 1053-1091. doi: 10.1086/655760

Schoon, Ingrid y Melis, Gabriella. (2019). Intergenerational transmission of family adversity: Examining constellations of risk factors. Journal PLoS ONE, 14(4), 1-18. https://doi.org/10.1371/journal.pone.0214801

Solís, Patricio. (2016). De joven a adulto en familia: trayectorias de emancipación familiar en México. En Marie-Laure Coubès, Patricio Solís y María Eugenia Zavala (Coords.), Generaciones, cursos de vida y desigualdad social en México (pp. 193-222). México: El Colegio de México, El Colegio de la Frontera Norte.

Solís, Patricio, Gayet, Cecilia y Juárez, Fátima. (2008). Las transiciones a la vida sexual, a la unión y a la maternidad en México: cambios en el tiempo y estratificación social. En Susana Lerner e Ivonne Szasz (Coords.), Salud reproductiva y condiciones de vida en México, Tomo I (pp. 397-428). México: El Colegio de México.

Stern, Claudio. (1997). El embarazo adolescente como problema público: una visión crítica. Salud Pública de México, 39(2), 137-143.

Stern, Claudio. (2007). Estereotipos de género, relaciones sexuales y embarazo adolescente en las vidas de jóvenes de diferentes contextos socioculturales en México. Estudios Sociológicos, 25(73), 105-129.

Stern, Claudio. (2012). El "problema" del embarazo en la adolescencia. Contribuciones a un debate. México: El Colegio de México.

Stern, Claudio y Menkes, Catherine. (2008). Embarazo adolescente y estratificación social. En Susana Lerner e Ivonne Szasz (Coords.), Salud reproductiva y condiciones de vida en México, Tomo I, (pp. 347-395). México: El Colegio de México. 
Szasz, Ivonne. (1997). Género y valores sexuales. Un estudio de caso entre un grupo de mujeres mexicanas. Estudios Demográficos y Urbanos, 12(1-2), 155-176. doi: http://dx.doi.org/10.24201/edu.v12i1.991

Szasz, Ivonne. (1998a). Masculine identity and the meanings of sexuality: a review of research in Mexico. Reproductive Health Matters, 6(12), 97-104. doi: https://doi.org/10.1016/S0968-8080(98)90012-X

Szasz, Ivonne. (1998b). Sexualidad y género: algunas experiencias de investigación en México. Debate feminista, $\quad 9(18), \quad$ 77-104. doi: https://doi.org/10.22201/cieg.2594066xe.1998.18.471

Szasz, Ivonne. (2001). La investigación sobre sexualidad y el debate sobre los derechos reproductivos en México. En José Gómez de León y Cecilia Rabell (Coords.), La población de México. Tendencias y perspectivas sociodemográficas hacia el siglo XXI (pp. 365-397). México: CONAPO, FCE.

Szasz, Ivonne. (2008). Relaciones de género y desigualdad socioeconómica en la construcción social de las normas sobre la sexualidad en México. En Susana Lerner e Ivonne Szasz (Coords.), Salud reproductiva y condiciones de vida en México (pp. 429-475). México: El Colegio de México.

Tuirán, Rodolfo. (1999). Dominios institucionales y trayectorias de vida en México. En Beatriz Figueroa (Coord.), México diverso y desigual. Enfoques sociodemográficos (pp. 207-241). México: El Colegio de México, Sociedad Mexicana de Demografía (SOMEDE).

Thomson, Rachel; Bell, Robert; Holland, Janet; Hendeson, Sheila; McGelliss, Sheena y Sharpe, Sue. (2002). Critical Moments: Choice, Change and Opportunity in Young People's Narratives of Transition. Sociology, 36(2), 335-354. doi: https://doi.org/10.1177/0038038502036002006 
Vandecasteele, Leen. (2010). Life Course Risks or Cumulative Disadvantage? The Structuring Effect of Social Stratification Determinants and Life Course Events on Poverty Transitions in Europe. European Sociological Review, 27(2), 246-263. doi: https://doi.org/10.1093/esr/jcq005

Wagmiller, Robert y Adelman, Robert. (2009). Childhood and Intergenerational Poverty. The Long-term Consequences of Growing up Poor. USA: Columbia University.

Welti, Carlos. (2000). Análisis demográfico de la fecundidad adolescente en México. Papeles de Población, 26, 43-87.

Welti, Carlos. (2005). Inicio de la vida sexual y reproductiva en México. Papeles de Población, 11(45), 143-176.

Zhu, Guangxing y Bosma, Alice. (2019). Early sexual initiation in Europe and its relationship with legislative change: A systematic review. International Journal of Law, Crime and Justice, 57, 70-82. doi: https://doi.org/10.1016/j.ijlcj.2019.03.001

\section{OLGA LORENA ROJAS}

Es doctora en Estudios de Población por El Colegio de México, institución donde labora como Profesora Investigadora. Miembro del Sistema Nacional de Investigadores en el nivel III. Sus temas de interés en la investigación están relacionados con el género, la vida familiar y la reproducción, con especial atención en el desempeño de los hombres en estos ámbitos. Temas sobre los que ha publicado diversos artículos en revistas especializadas, capítulos de libro, así como los libros Paternidad y vida familiar en la Ciudad de México y Estudios sobre la reproducción masculina. 